OPEN

SUBJECT AREAS:

GLYCOPROTEINS

GLYCOSYLATION

AGEING

SENESCENCE

Received

9 April 2013

Accepted

25 July 2013

Published

13 August 2013

Correspondence and requests for materials should be addressed to

G.J.F. (dianemch@ med.umich.edu)

\section{Age-dependent alterations of decorin glycosaminoglycans in human skin}

\author{
Yong Li, Ying Liu, Wei Xia, Dan Lei, John J. Voorhees \& Gary J. Fisher
}

Department of Dermatology, University of Michigan, Ann Arbor, Michigan.

Proteoglycans, a family of glycosaminoglycan (GAG) conjugated proteins, are important constituents of human skin connective tissue (dermis) and are essential for maintaining mechanical strength of the skin. Age-related alterations of dermal proteoglycans have not been fully elucidated. We quantified transcripts of 20 known interstitial proteoglycans in human skin and found that decorin was the most highly expressed. Decorin was predominantly produced by dermal fibroblasts. Decorin was localized in dermal extracellular matrix with GAG bound to type I collagen fibrils. Analysis of decorin extracted from young (21-30 years) and aged ( $>80$ years) sun-protected human buttock skin revealed that decorin molecular size in aged skin is significantly smaller than in young skin. The average size of decorin protein did not alter, indicating size of GAG chain is reduced in aged, compared to young skin. This age-dependent alteration of decorin GAG may contribute to skin fragility of elderly people.

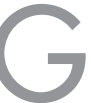

lycosaminoglycans (GAGs) are polysacchride chains composed of repeating dissacchride units. GAGs have high degrees of heterogeneity with regard to chain length and disaccharide composition ${ }^{1,2}$. GAGs comprise hyaluronic acids and are constituents of proteoglycans. Hyaluronic acids are composed of unsulfated and branched GAGs with molecular weights ranging from 10 to $10^{4} \mathrm{kDa}^{3}$. Hyaluronic acids function as ground substance to fill space in extracellular matrix (ECM), are particularly abundant in skin and joints. Proteoglycans consist of sulfated GAGs covalently linked to core proteins and have diverse localizations, such as cell surface, basement membrane and $\mathrm{ECM}^{4,5}$. Interstitial proteoglycans found in ECM can be classified into large aggregated proteoglycans (LAPs) and small leucine rich proteoglycans (SLRPs). LAPs consist of large core proteins (more than $100 \mathrm{kDa}$ ) and numerous GAGs and usually form large aggregates with hyaluronic acids. Four LAPs, including versican, aggrecan, brevican and neurocan are found in ECM of various connective tissues ${ }^{5}$. SLRPs form a growing, heterogeneous subfamily of proteoglycans, which are able to bind with a variety of proteins, including ECM proteins, particularly type I collagen ${ }^{4}$.

Decorin, the prototype of SLRP, is abundant in the dermal $\mathrm{ECM}^{6,7}$. Decorin comprises a single unbranched GAG linked to the N-terminal $4^{\text {th }}$ amino acid residue of core protein. Decorin core protein binds to specific locations on the surface of type I collagen fibrils and this binding is stabilized by electrostatic interaction of the GAG chain. Decorin binding is required for appropriate assembly of collagen fibrils and inhibits cleavage of collagen fibrils by matrix metalloprotease- $1^{8-10}$.

Decorin binding to type I collagen fibrils is thought to underlie the deleterious impact of decorin alterations on skin mechanical properties, which have been highlighted by studies in experimental animals and human diseases $^{7}$. Decorin deficiency is thought to be a pathogeneic factor for skin manifestiations, such as bruising and hyperelasticity, of Ehlers-Danlos syndrome, which is a group of inheritable diseases affecting connective tissues ${ }^{11}$. Alterations of decorin GAG are linked to the aged appearance seen in patients with a progeroid form of EhlersDanlos syndrome ${ }^{12-14}$. Both decorin null mice and genetically engineered mice with alterations in decorin GAG display skin fragility ${ }^{15-17}$.

Interestingly, skin fragility is also a characteristic of elderly individuals ${ }^{18}$. This observation promoted us to investigate potential age-dependent alterations of dermal interstitial proteoglycans $s^{6,19}$.

Although several proteoglycans, including decorin, biglycan and versican, have been found in dermal ECM ${ }^{20-25}$, a comprehensive and quantitative expression profile of interstitial proteoglycans in human skin has not been reported. In this study, we systematically characterized interstitial proteoglycans with respect to mRNA, protein and GAG in sun-protected skin of young (21-30 years old) and aged ( $>80$ years old) individuals. Our data demonstrate that decorin is the predominant type I collagen-binding proteoglycan in human dermis. In addition, our data indicate that size of decorin GAG is reduced in skin of elderly ( $>80$ years), compared to young (21-30 years). Alterations of decorin GAG likely contribute to skin fragility of elderly people. 


\section{Results}

Decorin is the predominant interstitial proteoglycan in human skin. In order to determine the gene expression profile, transcripts of all known 20 interstitial proteoglycans in full-thickness skin biopsies obtained from buttock of human subjects were quantified by realtime RT-PCR (Fig. 1a). We choose to study sun-protected buttock skin to minimize potential impact of sun exposure on gene expression. Transcripts of 11 proteoglycans, including aggrecan, brevican, epiphycan, keratocan, neurocan, nyctalopin, osteoglycin, opticin, podocan-like protein 1 (PODL-1), tsukushi-1 and tsukushi2 were not detected. Transcripts of 6 proteoglycans, including asporin, chondroadherin (CHAD), extracellular matrix protein 2 (EMP-2), osteomodulin, lumican and fibromodulin were near the limit of detection, approximately 1000-fold lower than expression of housekeeping gene 36B4.

mRNA expression of two SLRPs, decorin and biglycan; as well as one LAP, versican were much greater than the other detected proteoglycans. The level of decorin mRNA expression was similar to housekeeping gene 36B4 and was approximately 13-fold more than biglycan and 40 -fold more than versican. Relative levels of decorin, biglycan and versican transcripts were similar among sun-protected buttock, upper thigh and upper inner arm (Fig. 1b), suggesting that mRNA expression of these three proteoglycans is not specific to a single sun-protected anatomic location. The relative mRNA expression of decorin, biglycan and versican were further confirmed by realtime PCR utilizing commercial Taqman primers and probes from Applied Biosystems (data not shown), which yielded similar results as custom primers shown in Figure 1.

Decorin is produced primarily by dermal fibroblasts and decorin protein is localized in dermal ECM. We next investigated mRNA and protein localization of two most abundant skin interstitial proteoglycans, decorin and biglycan. Analysis of epidermis and dermis separated by laser capture microdissection showed that decorin mRNA was predominantly expressed in dermis, whereas biglycan mRNA levels in dermis and epidermis were similar (Fig. 2a).

We next separated fibroblasts from other dermal cells by collagenase-digestion of skin samples and fractionation using magnetic microbeads conjugated with antibody that recognizes a protein preferentially localized on the surface of fibroblasts (Miltenyi Biotech, Germany). Enrichment of fibroblasts was validated by quantification of mRNA expression of several cell markers. For instance, compared to fibroblast-depleted cells, fibroblast-enriched cells possessed approximately 100 -fold higher type I collagen mRNA. In contrast, bone marrow-derived cell marker CD45, endothelial cell marker CD31 and keratinocyte marker keratin-14 mRNA were undetectable in fibroblast-enriched cells, but were readily detectable in fibroblastdepleted cells (data not shown). Decorin mRNA was approximately 200 -fold higher in fibroblast-enriched cells than fibroblast-depleted cells (Fig. 2b). Biglycan expression levels tended to be relatively increased in fibroblasts, however, this trend was not statistically significant In situ hybridization showed that decorin mRNA was primarily localized in dermal stromal cells with fibroblast-like morphology, whereas biglycan mRNA was uniformly expressed by cells in both epidermis and dermis (Fig. 2c). Consistent with mRNA expression pattern, immunostaining showed that decorin protein was localized in dermal ECM, whereas biglycan protein was localized in both epidermis and dermis (Fig. 2d).

Decorin GAG intertwine with collagen fibrils. We next examined human skin specimens stained by cupromeronic blue, which preferentially binds to highly negatively charged sulfated GAGs in

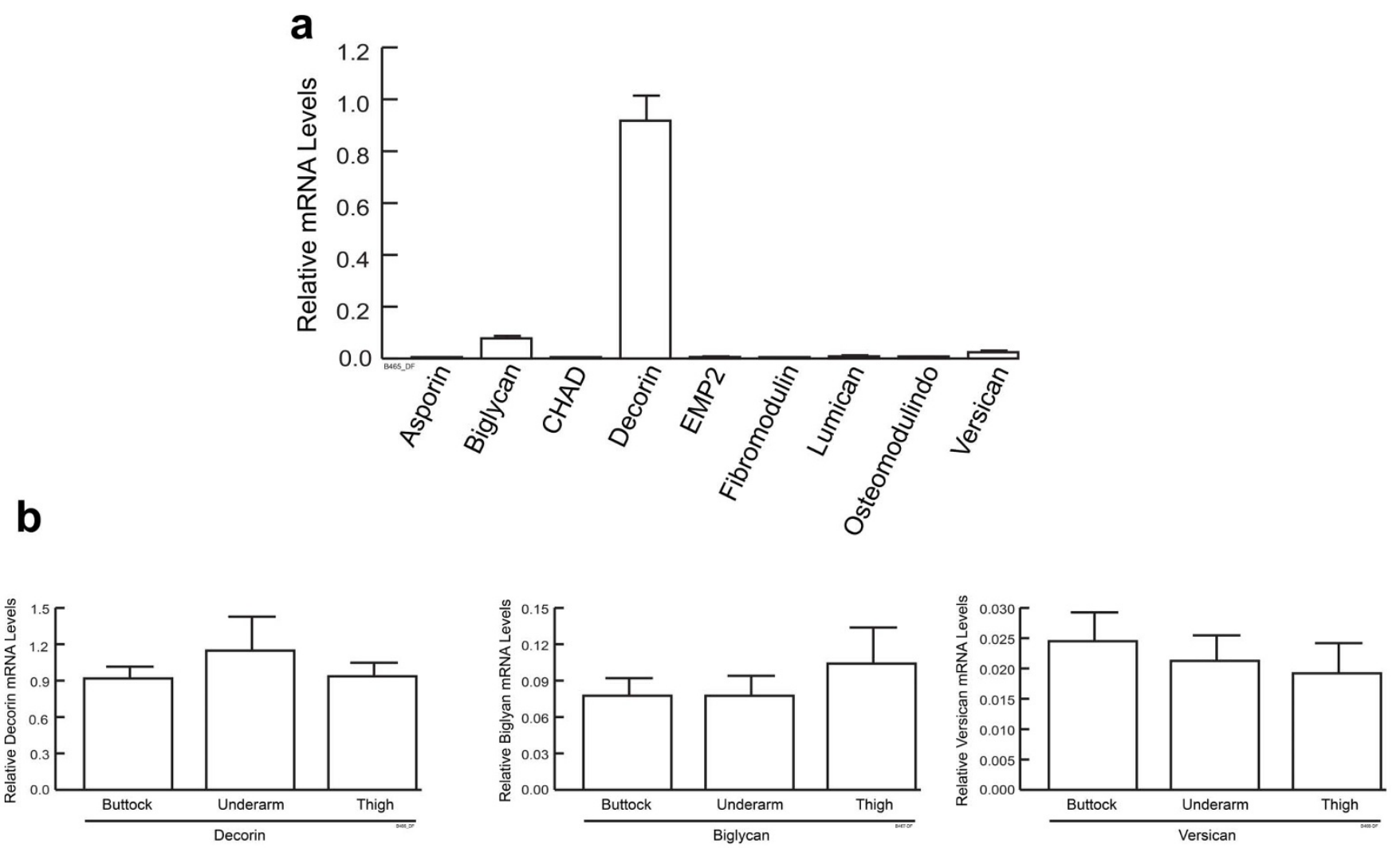

Figure $1 \mid$ Decorin is the most abundant interstitial proteoglycan in adult human skin. (a) Relative levels of proteoglycan transcripts in sun-protected buttock skin were determined by real-time RT-PCR. Transcripts for aggrecan, brevican, epiphycan, keratocan, neurocan, nyctalopin, osteoglycin, opticin, podocan-like protein 1 (PODL-1), tsukushi-1 and tsukushi-2 were not detected. $\mathrm{N}=3-10$. (b) Relative mRNA levels of decorin, biglycan and versican in skin of buttock $(\mathrm{N}=10)$, upper thigh $(\mathrm{N}=4)$ and upper inner arm $(\mathrm{N}=4)$. mRNA levels were normalized to housekeeping gene $36 \mathrm{~B} 4$. Results are mean + SEM. 
a

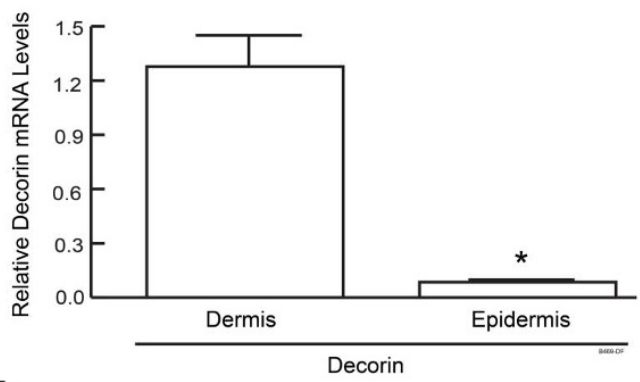

b

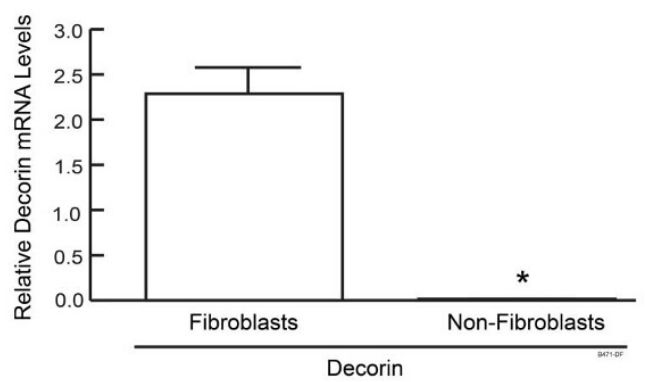

c Decorin Sense

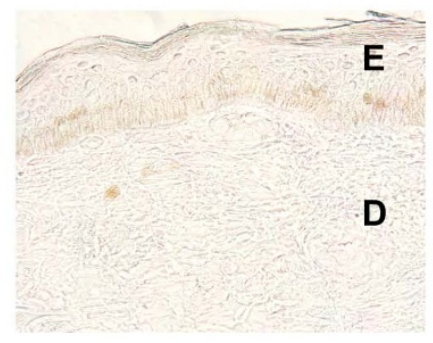

Biglycan Sense

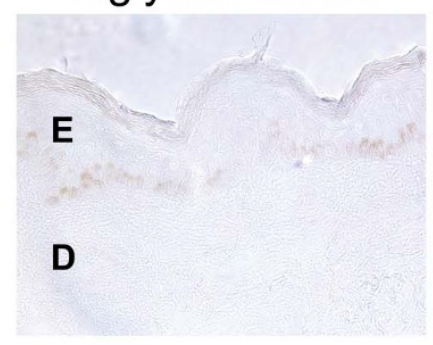

d

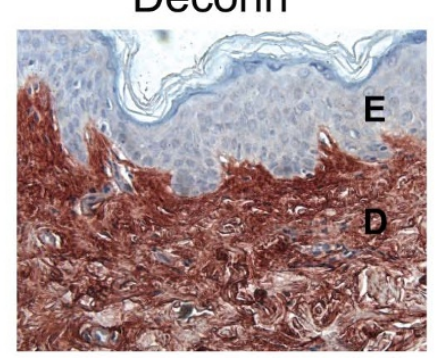

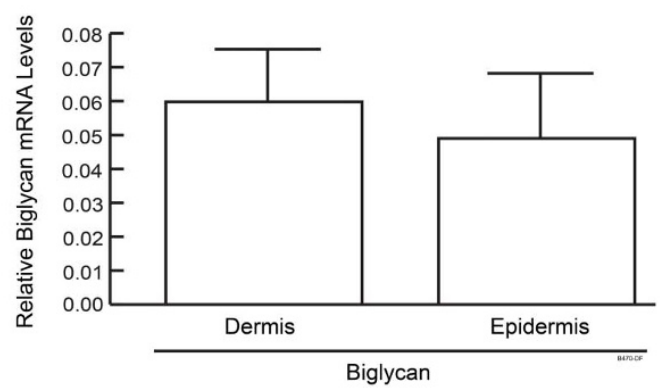

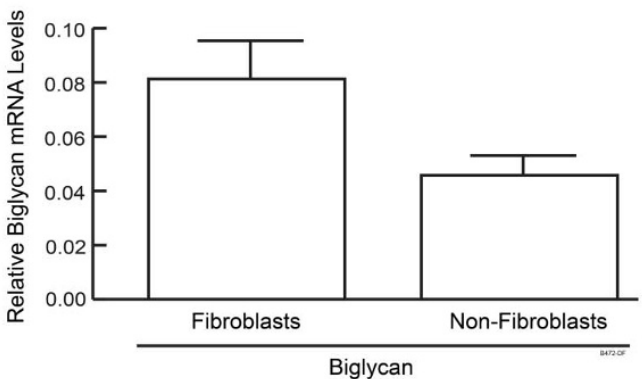

Decorin Anti-Sense
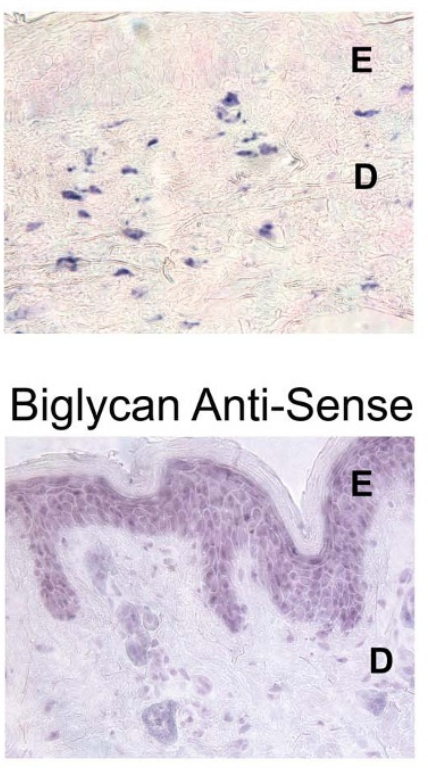

Biglycan

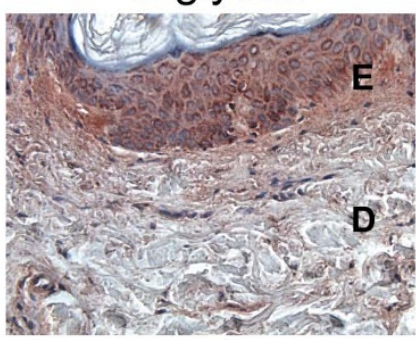

Figure $2 \mid$ Decorin is produced primarily by dermal fibroblasts in adult human skin (a) Epidermis and dermis of human skin specimens were separated by laser capture microdissection. Decorin and biglycan mRNA levels were determined by real-time RT-PCR and normalized to housekeeping gene 36B4. N $=6-7, * \mathrm{P}<0.01$. (b) Fibroblast-enriched cells and fibroblast-depleted cells were isolated from fresh adult buttock skin samples using Anti-Fibroblast Microbeads. Relative levels of decorin and biglycan mRNA were quantified by real time RT-PCR, $\mathrm{N}=4-6 * \mathrm{P}<0.01$, Results are mean + SEM.

(c) Decorin and biglycan transcripts were localized in human skin by in situ hybridization. Blue staining represents hybridized anti-sense probes. No specific staining was derived from sense probes. E: epidermis; D: dermis. Representative results from five human subjects are shown. (d) Immunostaining of decorin and biglycan in adult human skin. Results are representative of four human subjects. 
acidic $\mathrm{PH}$, using electron microscopy 9 . Stained GAGs appeared as electron dense circles or thin black filaments depending on GAG orientations in sections (Fig. 3). Individual collagen fibrils displayed as a whitish circles or rods with diameter approximately $60 \mathrm{~nm}$. Thorough examination of electron micrographs of dermis showed that most, if not all, collagen fibrils bound with GAGs. Collagen fibrils intertwined with GAGs comprised bulk of dermis.

In areas where collagen fibrils were tightly packed together, GAGs appeared as an orthogonal array on the surface of longitudinal sectioned collagen fibrils (Fig. 3a); consistent with this GAG- binding pattern, GAGs were arrayed around the circumstances of crosssectioned collagen fibrils (Fig. $3 \mathrm{~b}$ ). In areas where collagen fibrils were loosely packed, with larger inter-fibril distance, GAG chains formed inter-fibril bridges among nearby collagen fibrils, in addition to forming a ring around collagen fibrils (Fig. 3c). These bridges may provide mechanical coupling of the loosely packed fibrils. Chondroitinase, which degrades certain types of GAGs, including GAGs of decorin, biglycan and versican completely eliminated electron dense filaments surrounding collagen fibrils, confirming they represent GAGs (Fig. 3d). Collagen fibrils in close proximity to dermal stromal cells also were intertwined with GAGs (Fig. 3e). Given that decorin is the predominant interstitial proteoglycan localized in human dermis, it is likely that majority of collagen-binding GAGs are derived from decorin.

In contrast to well-organized pattern shown by collagen-binding GAGs, aggregated GAGs were observed in spaces between collagen fibrils (Fig. 3f). These GAGs were likely derived from space-filling LAPs. Since versican was the only LAP gene found to be detectably expressed in human skin, it is likely that these GAGs derive from versican.

Molecular size of decorin is reduced in aged, compared to young human skin. Western analysis using antibodies that specifically recognize $\mathrm{N}$-terminus, middle region or $\mathrm{C}$-terminus of decorin core protein revealed presence of both glycanated (core protein conjugated with GAG) and non-glycanated (core protein only) decorin in human skin (Fig. 4a and 4b). Existence of non-glycanated decorin was unlikely due to loss of GAGs during the extraction process, because extending incubation of skin biopsies with urea lysis buffer from 5 minutes to 16 hours did not increase the amount of non-glycanated decorin. Glycanated decorin appeared as a wide band between $64 \mathrm{kDa}$ to $110 \mathrm{kDa}$ in Western blot (Fig. $4 \mathrm{~b}$ and $4 \mathrm{c}$ ), due to heterogeneity of GAG chain lengths. Non-glycanated decorin appeared as several bands with molecular weights centered at approximately $50 \mathrm{kDa}$, due to heterogeneity of oligosaccharide chains. Chondroitinase converted glycanated decorin into non-glycanated decorin as previously reported ${ }^{26}$. Protein bands with molecular weights centered at approximately $100 \mathrm{kDa}$ detected in samples treated with chondroitinase were likely homodimers of non-glycanated decorin.

Buttock skin extracts obtained from young (21-30 years old) and aged ( $>80$ years old) individuals were normalized to total protein $(10 \mu \mathrm{g})$ and decorin was analyzed by Western blot (Fig. 4c). Comparing the wide bands in lanes without chondroitinase treatment, glycanated decorin from aged skin appeared to have molecular weights between $64 \mathrm{kDa}$ to $82 \mathrm{kDa}$; glycanated decorin from young skin appeared to have molecular weights between $64 \mathrm{kDa}$ to $100 \mathrm{kDa}$, indicating glycanated decorin from aged skin is substantially smaller compared to that of young skin. In contrast, comparing chondroitinase treated samples, molecular size of decorin core protein from both young and aged skin is approximately $50 \mathrm{kDa}$, suggesting molecular weights of decorin core protein do not differ between young and aged. Since sizes of decorin core protein are similar, the smaller size of glycanated decorin in aged skin is due to reduction of GAG sizes. Average molecular weight of decorin GAGs in aged skin appeared to be $8 \mathrm{kDa}$ smaller than in young skin ( $31 \mathrm{kDa}$ in aged vs. $39 \mathrm{kDa}$ in young skin, $\mathrm{N}=12, \mathrm{P}<0.05$ ).

In addition, Figure $4 \mathrm{c}$ shows that the amount of decorin core protein normalized to total protein amount did not significantly differ between young and aged human skin. However the amount of total sulfated GAGs, measured by Blyscan Assay, normalized to protein content was reduced by approximately $40 \%$ in aged skin compared to young skin (Fig. 5$)$. $(\mathrm{N}=10, \mathrm{P}<0.01)$. a

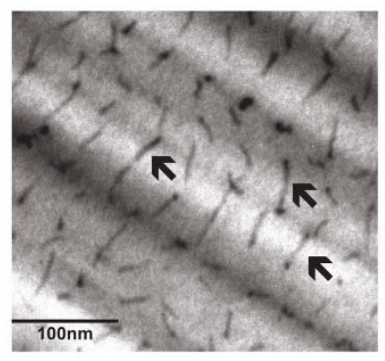

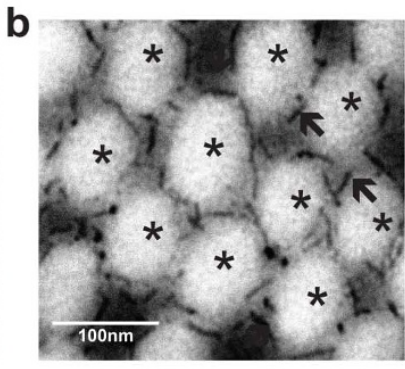

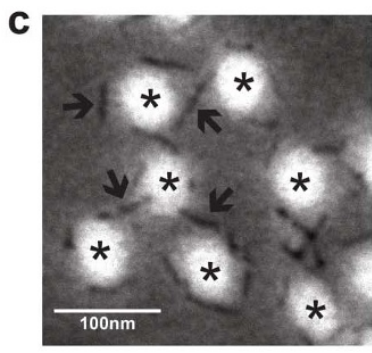

\section{d}

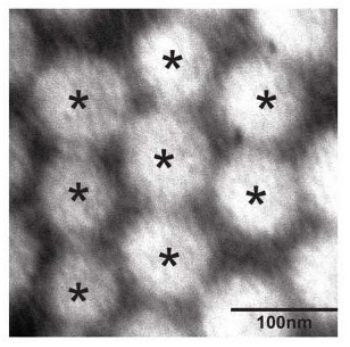

e

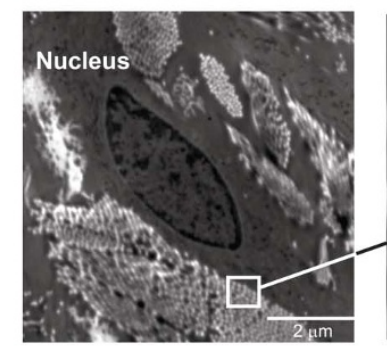

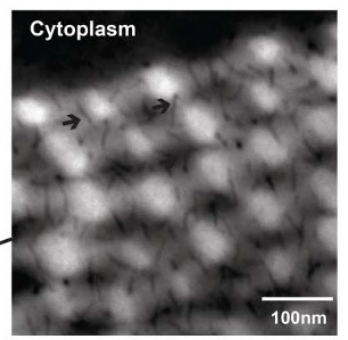

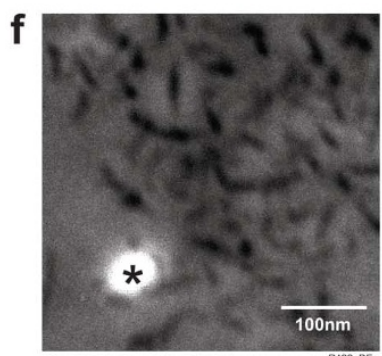

Figure 3 Ultrastructure of collagen fibril binding GAGs in human dermis. Sulfated GAGs were stained by cupromeronic blue and visualized using electron microscopy. GAGs displayed as black thin filaments are indicated by arrows. Collagen fibrils displayed as whitish rods or circles are indicated by asterisks. (a) Longitudinal sectioned collagen fibrils. (b) Cross-sectioned collagen fibrils. (c) Cross-sectioned loosely-packed collagen fibrils. (d) Skin specimens were treated with chondroitinase to degrade GAGs prior to cupromeronic blue staining. (e) A low magnification image of a spindle-shaped cell embedded in collagen fibrils and a high magnification image showing GAG-binding collagen fibrils associated with cell. (f) Aggregated GAGs localized in spaces between collagen fibrils. Results are representative of more than 200 micrographs of dermal ECM obtained from 5 human subjects. 
a

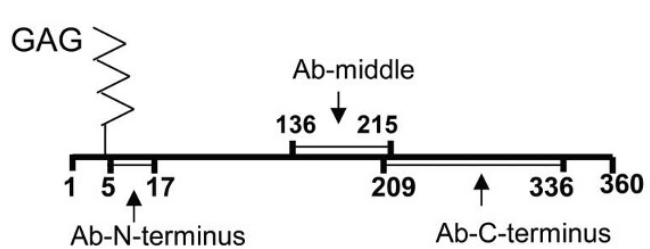

b

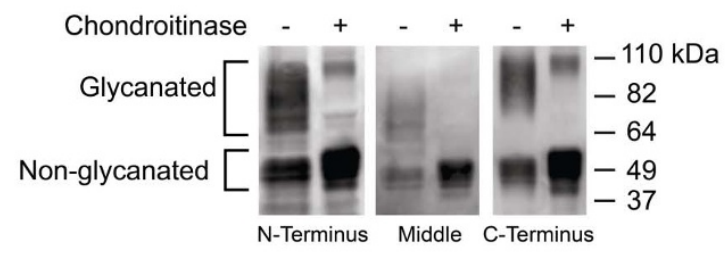

C

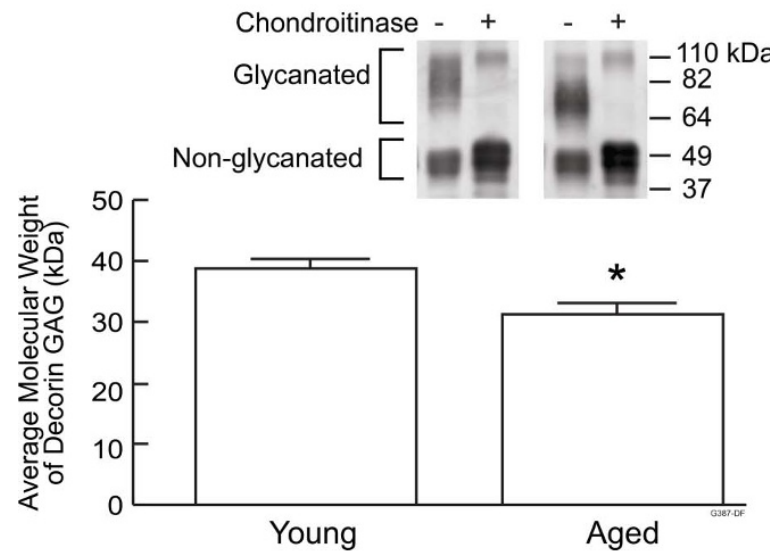

Figure $4 \mid$ Molecular size of decorin GAG is reduced in aged human skin. (a) Locations of epitopes of three anti-decorin antibodies. Numbers are amino acid positions within decorin core protein. (b) Adult human skin extracts were treated with or without chondroitinase to degrade GAGs and analyzed by Western blot using three anti-decorin antibodies, described in (a). Representative Western blots are shown, $\mathrm{N}=3$. Full-length blots are presented in Supplementary Figure 1a. (c) Skin extracts from young (2130 years old) and aged ( $>80$ years old) individuals were treated with or without chrondroitinase and analyzed by Western blots using antibody that recognizes C-terminal epitope. Bar graph shows average molecular weight of decorin GAG. Molecular weight of decorin GAG was calculated by subtraction of molecular weight of decorin core protein $(50 \mathrm{kDa})$ from average molecular weight of glycanated decorin. $\mathrm{N}=12$, ${ }^{*} \mathrm{P}<0.05$. Fulllength blots are presented in Supplementary Figure $1 \mathrm{~b}$.

\section{Discussion}

Quantitative gene expression of 20 known interstitial proteoglycans revealed that decorin, biglycan and versican are the most abundant in human skin. The protein expressions of these three proteoglycans in human skin in vivo were demonstrated by immunohistochemistry in this and previous studies ${ }^{27,28}$. Among these three proteoglycans, transcripts of decorin and biglycan, which are SLRPs, are greater than versican, which is a large aggregated proteoglycan. Electron microscopy revealed that the majority of sulfated GAGs in human dermis was directly associated with collagen fibrils, indicating they are collagen-binding SLRPs. These data are consistent with the finding that chondroitin sulfates, which is conjugated to versican, account for a small fraction of total sulfated GAGs in human $\operatorname{skin}^{29,30}$. The observation that decorin mRNA is the most abundant among all of the

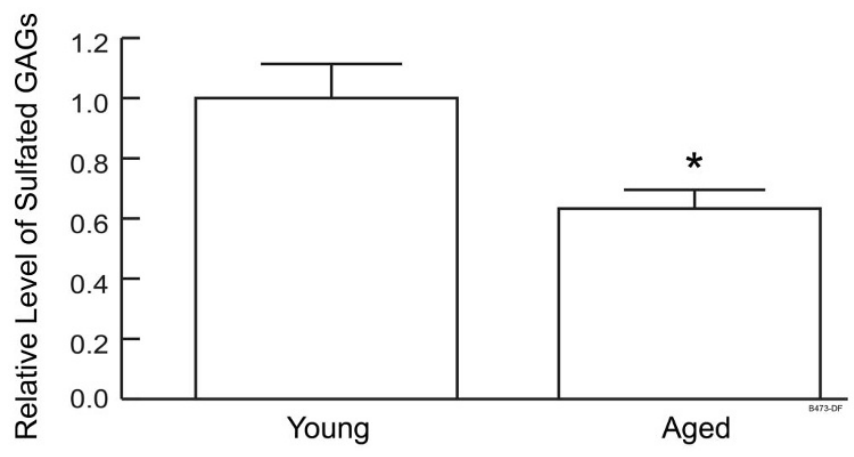

Figure 5 | Total sulfated GAGs are reduced in aged human skin. Sulfated GAGs in skin extracts from young and aged individuals were determined using Blyscan Sulfated Glycosaminoglycan Assay Kit and normalized to protein amount. $\mathrm{N}=10,{ }^{*} \mathrm{P}<0.05$.

proteoglycans examined is consistent with previous findings that dermatan sulfate, which is conjugated to decorin GAG, is the most abundant GAG in human skin ${ }^{30}$.

Although decorin and biglycan are both able to bind to type I collagen fibrils, their spatial expression in skin is strikingly different. Decorin is predominantly expressed by interstitial fibroblasts, consistent with its role as collagen-binding proteoglycan. In contrast, biglycan was expressed by both dermal and epidermal cells. This distinct expression pattern has also been observed in tissues other than skin ${ }^{5,31-33}$.

Given decorin's high abundance and restricted location in dermal ECM, it is likely that decorin comprises most, if not all, of type I collagen-binding proteoglycans in human skin. Studies of ultrastructure of GAGs in human skin revealed that decorin GAGs, bind to collagen fibrils in a periodic pattern similar to that described in skin of other mammals, such as rodent and bovine. These data support the concept that decorin plays critical roles in regulation of collagen fibril structure $^{9,34}$.

Evidence exists that molecular size of proteoglycan GAG, particularly decorin GAG, is controlled by pathophysiological conditions. For instance, decorin GAG size in tumor stroma, wound healing skin and hypertrophic scar is larger than normal tissues. Elongated decorin GAG chain is also described in embryonic compared to neonatal rats $^{35-37}$. Carrino et al, investigated decorin in skin biopsies obtained from several human subjects with age ranging from 20 to 56 years old and found that the major difference in decorin between younger and older individuals was reduced GAG length in older people ${ }^{6}$. In agreement with this work, we found evidence that size of decorin GAG is smaller in skin of the elderly. In addition, the amount of total sulfated GAGs is significantly less in aged human skin compared to young skin. Since our data indicate that decorin GAG comprises the majority of sulfated GAGs in human dermis, less total sulfated GAGs in aged human skin likely reflects lower decorin GAG amount. This lower amount likely results from the observed smaller molecular size of GAGs. In contrast, the abundance and size of decorin core protein are similar between young and aged human skin.

We have attempted to investigate mechanisms that regulate decorin GAG size in the context of skin aging. Evidence exists that fibroblasts in aged human skin have reduced responsiveness to TGF- $\beta^{18}$, and TGF-beta has been shown to enhance GAG size in cultured rat kidney fibroblast cell line, designated as $\mathrm{NRK}-49 \mathrm{~F}^{38}$. These observations prompted us to investigate the effects of TGF- $\beta$ on decorin synthesis in primary adult human skin fibroblasts. We found that TGF-beta treatment does not alter size of decorin secreted into culture medium, as demonstrated by Western analysis (supplemental Fig. 2). The differential effects of TGF- $\beta$ on decorin GAG length observed by us and the previous study ${ }^{38}$ might reflect differences in cell type used, i.e. primary human skin fibroblasts v.s. rat 
Table 1 | Real time PCR primer sequence

Gene

Primer sequence

Asporin-upstream

Asporin-downstream

Biglycan-upstream

Biglycan-downstream

CHAD-upstream

CHAD-downstream

Decorin-upstream

Decorin-downstream

Epiphycan-upstream

Epiphycan-downstream

EMP-upstream

EMP-downstream

Fibromodulin-upstream

Fibromodulin-downstream

Keratocan-upstream

Keratocan-downstream

Lumican-upstream

Lumican-downstream

Nyctalopin-upstream

Nyctalopin-downstream

Opticin-upstream

Opticin-downstream

Osteoglycin-upstream

Osteoglycin-downstream

Osteomodulin-upstream

Osteomodulin-downstream

Podocan-upstream

Podocan-downstream

Tsku l-upstream

Tskul-downstream

Tsku2-upstream

Tsku2-downstream

Versican-upstream

Versican-downstream gtccatttggatgtcagtgc

tcaagcattcgagtatcaaa

tctgtcacacccacctacagc

aggggagatctctttgggcac

ccttaccaataacccctgga

tgaacttggcaggtgaggca

cctgatgaccgcgacttcgag

tttggcactttgtccagaccc

taaggcaget cccagaattg

tcagtgaggt acagatgatg

acaccagtag caagacttcc

ccaaacagtg tcccttcttt

agtcaacaccaacctggagaa

cacctgcagcttggagaagtt

caaacctcag actgagacac

atagacctgcctcacacttc

tgagctggatctgtcctataa

atcttgcagaagctcttatg

atgaaaggec gagggatgtt

tccacggtgc tgcaggegca

cacgatgacc agacctacta

catcgcaata cacagaggaa

gatgaggcaa taacaccatt

ttggtaaggg tggtacagca

actcagtgtt ggacacaaca

gtgggtcaat agaaggacac

cgcctggttt ccattaggaa

ctgctgctcc tgctgitgcc

ctctatcaac tctcccacct

agcatggecg ggttgtctgg

aaaagcgctg gecggagggc

agcagcaggg gecacggcat

acgggattgaagacacacaag

agcctcaaaattcagtgtgta

kidney fibroblast cell line. In addition, we found that skin fibroblasts in cultures produce decorin with a very narrow size range centered around $110 \mathrm{kDa}$ (supplemental Fig. 2), which contrasts with the wide size range of decorin observed in human skin in vivo, suggesting metabolism of decorin differs significantly between in vivo and in vitro conditions. Thus, skin fibroblast cultures may not be a useful model to investigate the mechanisms controlling GAG size.

Decorin GAG size is theoretically determined by the balance between GAG elongation and degradation. The enzymatic process involved in GAG elongation has been elucidated. GAG polymerization occurs in Golgi compartment where repetitive disaccharide units are added to the decorin core protein via the enzymatic actions of specific glycosyltransferases ${ }^{39,40}$. Based on this process, numerous factors, such as the status of Golgi, the amount and activities of glycosyltransferases and the availability of disaccharides composed of iduronic acid and $N$-acetylgalactosamine, could potentially affect GAG polymerization, although empirical evidence is lacking. Investigations aimed at manipulation of GAG polymerization via altering these factors will help to understand how GAG synthesis is controlled and lay the foundation for exploring the underlying mechanisms of reduced decorin GAG size in elderly skin.

It is also possible that GAGs can be shortened post synthesis through enzymatic cleavage. In principle, decorin could be degraded in extracellular space where mature decorin is localized and/or inside lysosome followed by endocytosis ${ }^{41}$. The latter has been well demonstrated that fibroblasts take up extracellular decorin via receptormediated endocytosis, and subsequently deliver decorin to lysosomes where specific exoglycosidases, endoglycosidases and exosulfatases work in concert to degrade GAGs into monosacchride ${ }^{42}$. This intracellular degradation may not be directly relevant to

shortening of GAGs observed in aged human skin, as it normally results in complete decorin GAG degradation. Partial degradation of decorin GAGs via extracellular glycosidase activities during the aging process is a possible mechanism for age-dependent GAG shortening. However, all glycosidases identified to date are localized in lysosome, except for cell-membrane associated hyaluronoglucosaminidase, which degrades hyaluronic acid ${ }^{42}$. Glycosidases that could cleave decorin GAG in the extracellular space have yet to be identified.

Given the important role of decorin GAG in maintaining appropriate structure of collagen fibrils, alterations of decorin GAG likely influence collagen fibrils, and thus skin mechanical strength ${ }^{8,17}$. In addition, growing evidence suggest that decorin is involved in a wide range of cellular processes by regulating cellular signaling pathways via binding with various cytokines, growth factors and receptors through core protein and $\mathrm{GAG}^{43}$. Thus, alterations of decorin GAG may affect multiple aspects of skin biology.

\section{Methods}

Reagents. Plasmid containing decorin and biglycan cDNA were from Origene (Rockville, MD). Anti-biglycan antibody was from Abcam (Cambridge, MA). Antibody against N-terminal region of decorin was from Abnova (Walnut, CA). Antibody against middle region of decorin was from Santa Cruz (Santa Cruz, CA). Antibody against C-terminal region of decorin and Chondroitinase ABC were from Sigma (St. Louis, MO). RNA extraction kits were from Qiagen (Chatsworth, CA). Reagents for real-time PCR were from Applied Biosystems (Foster City, CA). Protein Assay Kit was from Bio-Rad Laboratories (Hercules, CA). Blyscan Sulfated Glycosaminoglycan Assay Kit was from Biocolor Ltd (United Kingdom). Collagenase and DIG RNA Labeling Kit were from Roche Applied Sciences (Germany). Antifibroblast Microbeads was from Miltenyi Biotec (Germany).

Ethics statement. This study was conducted in compliance with Declaration of Helsinki principles. All procedures involving human subjects were approved by the University of Michigan Institutional Review Board, and informed written consent was obtained from all human subjects.

Human tissue procurement. Whole punch skin biopsies (4 $\mathrm{mm}$ or $6 \mathrm{~mm}$ in diameter) were taken from buttock, underarm and upper thigh of healthy human subjects as previously described ${ }^{44}$. Skin biopsies were immediately embedded in optimal cutting temperature compound (OCT, Tissue-Tek, Miles Inc., Elkhart, IA), snap frozen in liquid nitrogen, and stored at $-80^{\circ} \mathrm{C}$ as previously described ${ }^{44}$.

Laser capture microdissection (LCM). LCM was performed as previously described ${ }^{45}$. Briefly, human skin punch biopsies embedded in OCT were sectioned. Dermis and epidermis were captured using LCM (Leica ASLMD system; Leica Microsystems, Wetzlar, Germany) and stored in $-86^{\circ} \mathrm{C}$ until RNA extraction.

RNA extraction and real-time RT-PCR. Epidermis and dermis obtained from LCM and full-thickness skin biopsies underwent RNA extraction and subsequent real-time RT-PCR as described previously ${ }^{40,41}$. The primers for real-time PCR were listed in Table $1^{46,47}$

Isolation of fibroblast-enriched cells from skin biopsies. Skin biopsies $(6 \mathrm{~mm}$ in diameter) were minced into small pieces and then digested by collagenase $(5 \mathrm{mg} / \mathrm{ml}$ in DMEM) for 16 hours at $37^{\circ} \mathrm{C}$ as described previously ${ }^{48}$. Cells and tissue debris obtained from digested skin biopsies were filtered by 100-micron filters. Fibroblasts were separated from non-fibroblast cells using Anti-fibroblast Microbeads (Miltenyi Biotec) according to manufacturer's instruction.

Proteoglycan extraction and Western blot analysis. Interstitial proteoglycan were extracted from skin biopsies using urea lysis buffer as previously described ${ }^{26}$. Briefly, skin biopsy embed in OCT were cut into 20 micron sections. Skin specimens (total 500 micron) were washed with phosphate buffered saline (PBS) to remove OCT, and immediately lysed in $300 \mu \mathrm{l}$ urea lysis buffer (50 mM Tris, PH 7.4, urea 7 M, $150 \mathrm{mM}$ $\mathrm{NaCl}, 1 \%$ Triton, proteinase inhibitor cocktail (Roche)). Skin extracts were agitated at $4{ }^{\circ} \mathrm{C}$ for 20 minutes and spun at $12,000 \mathrm{rpms}$ for 10 minutes. Supernatants were dialyzed against PBS to remove urea. Supernatants (containing $10 \mu \mathrm{g}$ protein) were analyzed by SDS-PAGE electrophoresis and Western Blot. In some experiments, lysates were treated with chondroitinase $\operatorname{ABC}(0.2 \mathrm{unit} / \mathrm{ml})$ for 2 hours at $37^{\circ} \mathrm{C}$. Western blots were performed using anti-decorin antibodies and secondary antibody conjugated with alkaline phosphatase. The blots were developed using chemifluorescence substrate for alkaline phosphatase (GE Healthcare, Piscataway, NJ). Chemifluorescence was detected by Storm Molecular-Imager (GE Healthcare) and quantified by Image Quant software.

Immunohistochemistry. Immunostaining was performed using anti-decorin, antibiglycan and non-immune antibodies as previously described ${ }^{49}$. In all cases, antidecorin and anti-biglycan antibodies were used at concentrations and fixation conditions that yielded no observable nonspecific staining. Sections were 
counterstained with hematoxylin. Stained tissue sections were photographed using a Zeiss microscope (Axioskop 2) and images were obtained with digital camera (SPOT2, Diagnostic Inc. Sterling Heights, MI).

In situ hybridization. In situ hybridization was performed using single-strand RNA probes comprising antisense or sense sequences of decorin or biglycan. Digoxigenin (DIG)-labeled RNA probes were synthesized by in vitro transcription with T7 polymerases using a DIG RNA Labeling Kit (Roche). Frozen skin sections (5 mm) were fixed and hybridized with RNA probes as previously described ${ }^{50}$. Hybridization signals were detected immunohistochemically by alkaline phosphatase-conjugated anti-DIG antibody.

Electron microscopy. Sulfated GAGs were stained by cupromeronic blue as previously described ${ }^{9}$. Briefly, fresh skin biopsies were cut into sections with approximately $1 \mathrm{~mm}$ in thickness and immediately fixed in fixation solution $(2.5 \%$ (w/v) glutaraldehyde, $25 \mathrm{mM}$ acetic acid and $25 \mathrm{mM}$ sodium acetate ( $\mathrm{pH} 5.8)$ ) overnight at $4{ }^{\circ} \mathrm{C}$. The next day, specimens were washed with buffer $(25 \mathrm{mM}$ acetic acid and $25 \mathrm{mM}$ sodium acetate ( $\mathrm{pH}$ 5.8)). Specimens were stained with staining buffer $\left(0.05 \%(\mathrm{w} / \mathrm{v})\right.$ cupromeronic blue, $0.1 \mathrm{M} \mathrm{MgCl}_{2}$ and $25 \mathrm{mM}$ sodium acetate ( $\mathrm{pH} 5.8), 2.5 \%$ glutaraldehyde $(\mathrm{w} / \mathrm{v})$ ) for 2 hours at room temperature. Specimens were further incubated with $0.034 \mathrm{M} \mathrm{Na}_{2} \mathrm{WO}_{4}$ for one hour at room temperature. After staining, specimens were dehydrated in graded ethanol, embedded and cut into ultra-thin sections. Sections were analyzed by transmission electron microscopy at the Microscopy Image Laboratory, University of Michigan.

Quantification of total sulfated GAGs. Buttock skin biopsies obtained from young (21-30 years old) and aged ( $>80$ years old) individuals were cut into 20 -micron sections and lysed in urea lysis buffer as described above. Skin extracts, containing $300 \mu \mathrm{g}$ protein, were incubated with papain proteinase at $65^{\circ} \mathrm{C}$ for 3 hours. After proteinase digestion, sulfated GAGs were quantified by Blyscan Sulfated Glycosaminoglycan Assay Kit (Biocolor Ltd) according to the manufacturer's instruction.

1. Gandhi, N. S. \& Mancera, R. L. The structure of glycosaminoglycans and their interactions with proteins. Chem Biol Drug Des 72, 455-482 (2008)

2. van Lis, J. glycosaminoglycans in human skin. Br J Dermatol 88, 355-361 (1973).

3. Meyer, L. J. \& Stern, R. Age-dependent changes of hyaluronan in human skin. J Invest Dermatol 102, 385-389 (1994)

4. Dellett, M., Hu, W., Papadaki, V. \& Ohnuma, S. Small leucine rich proteoglycan family regulates multiple signalling pathways in neural development and maintenance. Dev Growth Differ 54, 327-340 (2012).

5. Iozzo, R. V. Matrix proteoglycans: from molecular design to cellular function. Annu Rev Biochem 67, 609-652 (1998).

6. Carrino, D. A. et al. Age-related differences in human skin proteoglycans. Glycobiology 21, 257-268 (2011)

7. Seidler, D. G. The galactosaminoglycan-containing decorin and its impact on diseases. Curr Opin Struct Biol 22, 578-582 (2012).

8. Ruhland, C. et al. The glycosaminoglycan chain of decorin plays an important role in collagen fibril formation at the early stages of fibrillogenesis. FEBS J 274, 4246-4255 (2007).

9. Scott, J. E. Proteoglycan: collagen interactions in connective tissues. Ultrastructural, biochemical, functional and evolutionary aspects. Int J Biol Macromol 13, 157-161 (1991).

10. Stuart, K., Paderi, J., Snyder, P. W., Freeman, L. \& Panitch, A. Collagen-binding peptidoglycans inhibit MMP mediated collagen degradation and reduce dermal scarring. PLoS One 6, e22139 (2011).

11. Wu, J., Utani, A., Endo, H. \& Shinkai, H. Deficiency of the decorin core protein in the variant form of Ehlers-Danlos syndrome with chronic skin ulcer. J Dermatol Sci 27, 95-103 (2001).

12. Beavan, L. A. et al. Deficient expression of decorin in infantile progeroid patients. J Biol Chem 268, 9856-9862 (1993).

13. Furukawa, K. \& Okajima, T. Galactosyltransferase I is a gene responsible for progeroid variant of Ehlers-Danlos syndrome: molecular cloning and identification of mutations. Biochim Biophys Acta 1573, 377-381 (2002).

14. Gotte, M. \& Kresse, H. Defective glycosaminoglycan substitution of decorin in a patient with progeroid syndrome is a direct consequence of two point mutations in the galactosyltransferase I (beta4GalT-7) gene. Biochem Genet 43, 65-77 (2005).

15. Danielson, K. G. et al. Targeted disruption of decorin leads to abnormal collagen fibril morphology and skin fragility. J Cell Biol 136, 729-743 (1997).

16. Jungmann, O. et al. The dermatan sulfate proteoglycan decorin modulates alpha2beta1 integrin and the vimentin intermediate filament system during collagen synthesis. PLoS One 7, e50809 (2012).

17. Maccarana, M. et al. Dermatan sulfate epimerase 1-deficient mice have reduced content and changed distribution of iduronic acids in dermatan sulfate and an altered collagen structure in skin. Mol Cell Biol 29, 5517-5528 (2009).

18. Fisher, G. J., Varani, J. \& Voorhees, J. J. Looking older: fibroblast collapse and therapeutic implications. Arch Dermatol 144, 666-672 (2008).

19. Oh, J. H., Kim, Y. K., Jung, J. Y., Shin, J. E. \& Chung, J. H. Changes in glycosaminoglycans and related proteoglycans in intrinsically aged human skin in vivo. Exp Dermatol 20, 454-456 (2011).
20. Carrino, D. A., Mesiano, S., Barker, N. M., Hurd, W. W. \& Caplan, A. I. Proteoglycans of uterine fibroids and keloid scars: similarity in their proteoglycan composition. Biochem J 443, 361-368 (2012).

21. Fleischmajer, R. et al. Decorin interacts with fibrillar collagen of embryonic and adult human skin. J Struct Biol 106, 82-90 (1991).

22. Hasegawa, K. et al. Versican, a major hyaluronan-binding component in the dermis, loses its hyaluronan-binding ability in solar elastosis. J Invest Dermatol 127, 1657-1663 (2007).

23. Honardoust, D., Varkey, M., Marcoux, Y., Shankowsky, H. A. \& Tredget, E. E. Reduced decorin, fibromodulin, and transforming growth factor-beta3 in deep dermis leads to hypertrophic scarring. J Burn Care Res 33, 218-227 (2012).

24. Lundqvist, K. \& Schmidtchen, A. Immunohistochemical studies on proteoglycan expression in normal skin and chronic ulcers. Br J Dermatol 144, 254-259 (2001).

25. Malgouries, S., Thibaut, S. \& Bernard, B. A. Proteoglycan expression patterns in human hair follicle. Br J Dermatol 158, 234-242 (2008).

26. Carrino, D. A., Sorrell, J. M. \& Caplan, A. I. Age-related changes in the proteoglycans of human skin. Arch Biochem Biophys 373, 91-101 (2000).

27. Bernstein, E. F. et al. Differential expression of the versican and decorin genes in photoaged and sun-protected skin. Comparison by immunohistochemical and northern analyses. Lab Invest 72, 662-669 (1995).

28. Werth, B. B., Bashir, M., Chang, L. \& Werth, V. P. Ultraviolet irradiation induces the accumulation of chondroitin sulfate, but not other glycosaminoglycans, in human skin. PLoS One 6, e14830 (2011).

29. Flint, M. H., Craig, A. S., Reilly, H. C., Gillard, G. C. \& Parry, D. A. Collagen fibril diameters and glycosaminoglycan content of skins-ndices of tissue maturity and function. Connect Tissue Res 13, 69-81 (1984).

30. Takahashi, Y. et al. Disaccharide analysis of human skin glycosaminoglycans in sun-exposed and sun-protected skin of aged people. J Dermatol Sci 11, 129-133 (1996).

31. Schaefer, L. et al. The matrix component biglycan is proinflammatory and signals through Toll-like receptors 4 and 2 in macrophages. J Clin Invest 115, 2223-2233 (2005).

32. Ungefroren, H. \& Krull, N. B. Transcriptional regulation of the human biglycan gene. J Biol Chem 271, 15787-15795 (1996).

33. Yamamoto, K. et al. Biglycan is a specific marker and an autocrine angiogenic factor of tumour endothelial cells. Br J Cancer 106, 1214-1223 (2012).

34. Reed, C. C. \& Iozzo, R. V. The role of decorin in collagen fibrillogenesis and skin homeostasis. Glycoconj J 19, 249-255 (2002).

35. Kuwaba, K., Kobayashi, M., Nomura, Y., Irie, S. \& Koyama, Y. Elongated dermatan sulphate in post-inflammatory healing skin distributes among collagen fibrils separated by enlarged interfibrillar gaps. Biochem J 358, 157-163 (2001).

36. Nomura, Y. Structural change in decorin with skin aging. Connect Tissue Res 47, 249-255 (2006)

37. Garg, H. G., Siebert, J. W., Garg, A. \& Neame, P. J. Iduronic acid-rich proteoglycans (PGIdoA) and human post-burn scar maturation: isolation and characterization. Carbohydr Res 267, 105-113 (1995).

38. Bassols, A. \& Massague, J. Transforming growth factor beta regulates the expression and structure of extracellular matrix chondroitin/dermatan sulfate proteoglycans. J Biol Chem 263, 3039-3045 (1988).

39. Izumikawa, T. et al. Identification of chondroitin sulfate glucuronyltransferase as chondroitin synthase- 3 involved in chondroitin polymerization: chondroitin polymerization is achieved by multiple enzyme complexes consisting of chondroitin synthase family members. J Biol Chem 283, 11396-11406 (2008).

40. Kjellen, L. \& Lindahl, U. Proteoglycans: structures and interactions. Annu Rev Biochem 60, 443-475 (1991).

41. Feugaing, D. D. et al. Endocytosis of the dermatan sulfate proteoglycan decorin utilizes multiple pathways and is modulated by epidermal growth factor receptor signaling. Biochimie 89, 637-657 (2007).

42. Silbert, J. E. Structure and metabolism of proteoglycans and glycosaminoglycans. $J$ Invest Dermatol 79 Suppl 1, 31s-37s (1982).

43. Neill, T., Schaefer, L. \& Iozzo, R. V. Decorin: a guardian from the matrix. Am Pathol 181, 380-387 (2012).

44. Li, Y., Liu, Y., Xu, Y., Voorhees, J. J. \& Fisher, G. J. UV irradiation induces Snail expression by AP-1 dependent mechanism in human skin keratinocytes. J Dermatol Sci 60, 105-113 (2010)

45. Fisher, G. J. et al. Collagen fragmentation promotes oxidative stress and elevates matrix metalloproteinase-1 in fibroblasts in aged human skin. Am J Pathol 174, 101-114 (2009)

46. Quan, T., He, T., Kang, S., Voorhees, J. J. \& Fisher, G. J. Ultraviolet irradiation alters transforming growth factor beta/smad pathway in human skin in vivo. J Invest Dermatol 119, 499-506 (2002).

47. Quan, T., He, T., Kang, S., Voorhees, J. J. \& Fisher, G. J. Solar ultraviolet irradiation reduces collagen in photoaged human skin by blocking transforming growth factor-beta type II receptor/Smad signaling. Am J Pathol 165, 741-751 (2004)

48. Zaba, L. C., Fuentes-Duculan, J., Steinman, R. M., Krueger, J. G. \& Lowes, M. A. Normal human dermis contains distinct populations of CD11c+BDCA-1+ dendritic cells and CD163+FXIIIA + macrophages. J Clin Invest 117, 2517-2525 (2007).

49. Fisher, G. J. et al. Ultraviolet irradiation increases matrix metalloproteinase-8 protein in human skin in vivo. I Invest Dermatol 117, 219-226 (2001). 
50. Quan, T., He, T., Kang, S., Voorhees, J. J. \& Fisher, G. J. Connective tissue growth factor: expression in human skin in vivo and inhibition by ultraviolet irradiation. J Invest Dermatol 118, 402-408 (2002).

\section{Acknowledgements}

We would like to thank Suzan Rehbine, LPN, for her help with volunteer recruitment and procurement of biopsies. We would like to thank Diane Fiolek for her help with graphics and editing of manuscript.

\section{Author contributions}

Y.Li, G.J.F. and J.J.V. development of conceptual framework, design of experiments. Y.Li and G.F. data analysis and manuscript writing. Y.Liu, W.X. and D.L. performed experiments that generated data described in the text. All authors reviewed the manuscript.

\section{Additional information}

Supplementary information accompanies this paper at http://www.nature.com/ scientificreports

Competing financial interests: The authors declare no competing financial interests.

How to cite this article: Li, Y. et al. Age-dependent alterations of decorin glycosaminoglycans in human skin. Sci. Rep. 3, 2422; DOI:10.1038/srep02422 (2013).

(C) $\Theta$ This work is licensed under a Creative Commons Attributioncc. visit http://creativecommons.org/licenses/by-nc-nd/3.0 